\title{
Serum Tenascin-X Strongly Binds to Vascular Endothelial Growth Factor
}

\author{
Taichi Ishitsuka, ${ }^{a}$ Tomoki Ikuta, ${ }^{a}$ Hiroyoshi Ariga, ${ }^{a}$ and Ken-ichi Matsumoto ${ }^{*}, a, b$ \\ ${ }^{a}$ Department of Molecular Biology, Graduate School of Pharmaceutical Sciences, Hokkaido University; Kita-ku, Sapporo \\ 060-0812, Japan: and ${ }^{b}$ Department of Biosignaling and Radioisotope Experiment, Center for Integrated Research in \\ Science, Shimane University; Enya-cho, Izumo 693-8501, Japan. \\ Received January 20, 2009; accepted March 23, 2009; published online March 27, 2009
}

Interstitial extracellular matrix tenascin-X (iTNX) with about $450 \mathrm{kDa}$ is prominently present in various tissues. Previously, we identified the serum form of TNX (sTNX) with $200 \mathrm{kDa}$ in the mouse. In the present study, in order to investigate distinctive features and functions of sTNX, a plasmid encoding the recombinant mouse sTNX was constructed. As a control, we also constructed a plasmid encoding mouse 450-kDa iTNX and a plasmid encoding 250-kDa iTNX, which lacks the region of 200-kDa sTNX from 450-kDa iTNX. In cells stably expressing each recombinant TNX, a more than 7-fold larger amount of 200-kDa sTNX was released into conditioned medium than the amounts of $250-\mathrm{kDa}$ iTNX and $450-\mathrm{kDa}$ iTNX released into the medium. We previously reported that a splice isoform of iTNX (340-kDa iTNX) binds to vascular endothelial growth factor B (VEGF-B) as well as to VEGF-A. Therefore, the ability of VEGF-A and VEGF-B to bind to 200-kDa sTNX was examined by a co-immunoprecipitation assay in comparison with the binding abilities to 250-kDa iTNX and 450-kDa iTNX. It was found that sTNX strongly bound to VEGF-A and VEGF-B, compared with the binding abilities of other iTNX proteins. Based on the results of assays of incorporation of 5-ethynyl-2'-deoxyuridine (EdU), we found that purified recombinant 200-kDa sTNX both alone and in combination with VEGF-A or basic fibroblast growth factor (bFGF) can weakly promote DNA synthesis in proliferating vascular endothelial cells (UV 92 cells). These results suggest that STNX possesses weak activity for proliferation of endothelial cells.

Key words tenascin-X; extracellular matrix; serum form; vascular endothelial growth factor

The extracellular matrix (ECM) was originally thought to be merely a physical framework for cells in terms of mechanical strength. However, this concept has been revised in recent years. The ECM is now known to participate in cell proliferation, migration, differentiation, and survival. ${ }^{1)}$ The ECM contains not only many adhesive proteins, including fibronectin, collagen and laminin, which generally promote cell attachment or migration, but also adhesive modulatory proteins such as tenascin, thrombospondin and SPARC/ osteonectin/BM40, which regulate the interactions between cell receptors and adhesive proteins.

Among adhesive modulatory proteins, the tenascin family constitutes a group of ECM glycoproteins with a characteristic structure. The four members of this family identified so far [tenascin-C (TNC), restrictin/J1-160/180 (tenascin$\mathrm{R}$, TNR), tenascin-X (TNX), and tenascin-W/tenascin-N (TNW/TNN)] have been found in vertebrates. ${ }^{2-6)}$ Tenascin family members are made up of the same types of structural domains, including a cysteine-rich segment at the amino terminus, epidermal growth factor (EGF)-like repeats, fibronectin type III (FNIII)-like repeats, and a fibrinogen-like domain at the carboxyl terminus.

TNX is the largest member of the tenascin family. Complete deficiency of TNX in humans leads to a rare recessive form of Ehlers-Danlos Syndrome (EDS), and TNX haploinsufficiency is associated with hypermobility type of EDS. The skin of TNX-deficient patients is markedly lax with poor recoil properties and shows easy bruising. ${ }^{7-9)}$ In the skin of these patients, the collagen density is reduced in the dermis, and the elastic fibers are abnormal. Furthermore, recent genetic studies have revealed a strong association between the TNX locus and other diseases such as schizophrenia, ${ }^{10)}$ arterial tortuosity syndrome (ATS), ${ }^{11)}$ and systemic lupus erythematosus. ${ }^{12)}$ Recent evidence has suggested that TNX is in- volved in collagen fibrillogenesis, ${ }^{13,14)}$ collagen deposition, ${ }^{15)}$ and development and maintenance of elastic fibers. ${ }^{16}$ ) As a biological feature of TNX, it has been shown that TNX interacts with types I, III and V fibrillar collagen and fibril-associated types XII and XIV collagen ${ }^{15,17)}$ and with proteoglycan decorin. $^{18)}$

Schalkwijk et ll $^{8)}$ reported that human TNX is also present in normal serum with an apparent molecular size of $140 \mathrm{kDa}$. Measurement of serum TNX revealed that high levels of serum TNX are a risk factor for abdominal aortic aneurysm. ${ }^{19)}$ We previously identified the serum form of TNX (referred to as sTNX) with a molecular mass of $200 \mathrm{kDa}$ in the mouse, ${ }^{20)}$ and we gave the name iTNX to conventional interstitial TNX. The full-length iTNX has a molecular mass of about $450 \mathrm{kDa}$. The $200-\mathrm{kDa}$ sTNX contains the last 15 FNIII repeats and a fibrinogen domain identical to the C-terminal portion of the $450-\mathrm{kDa}$ iTNX. The N-terminus of sTNX is located in the juncture between the 16th FNIII and 17th FNIII repeats. Furthermore, 200-kDa sTNX is generated by proteolytic cleavage of the $450-\mathrm{kDa}$ iTNX.

A number of fragments of ECM molecules have been identified as angiogenesis inhibitors. Angiostatin, a $38-\mathrm{kDa}$ proteolytic fragment of plasminogen containing the first fourkringle structures, inhibits proliferation, blocks migration, and increases apoptosis of endothelial cells. ${ }^{21)}$ Likewise, endostatin, the 20-kDa C-terminal fragment of the NC1 domain of the $\alpha 1$ chain of type XVIII collagen is a potent inhibitor of angiogenesis. ${ }^{22)}$ Endostatin specifically inhibits endothelial proliferation and potently inhibits angiogenesis and tumor growth. Canstatin ${ }^{23)}$ and tumstatin ${ }^{24)}$ derived from the $\mathrm{NC1}$ domain of type IV collagen have also been shown to have anti-angiogenic activities. Besides the derivatives from collagen, anastellin, a 76-aa peptide derived from the first type III repeat in fibronectin, ${ }^{25)}$ and endorepellin, the $\mathrm{C}$ terminus of 
perlecan (a ubiquitous basement membrane heparan sulfate proteoglycan), ${ }^{26}$ have been reported to inhibit angiogenesis. These endogenous angiogenesis inhibitors have significant therapeutic potential.

We previously have demonstrated that a splice isoform of iTNX that lacks the 3rd FNIII repeat (M3) and the 15th FNIII to the 22nd FNIII repeat (M15-M22), 340-kDa iTNX, binds to vascular endothelial growth factor B (VEGFB) as well as VEGF-A and that VEGF-B in combination with $340-\mathrm{kDa}$ iTNX strongly promotes DNA synthesis in endothelial cells compared with the effect of VEGF-B alone. ${ }^{27)}$ Furthermore, since $200-\mathrm{kDa}$ sTNX is derived from the C-terminal portion of $450-\mathrm{kDa}$ iTNX, we were interested to clarify whether sTNX is involved in the proliferation of endothelial cells. For this end, in this study we examined distinctive features of sTNX such as susceptibility to be released into conditioned medium, binding capability to VEGF-A and VEGF-B, and proliferation activity for endothelial cells.

\section{MATERIALS AND METHODS}

Cell Cultures Human embryo kidney $293 \mathrm{~T}$ cells were cultured at $37^{\circ} \mathrm{C}$ in a $5 \% \mathrm{CO}_{2}$ humidified atmosphere in Dulbecco's modified Eagle's medium (DMEM) (Nissui Pharmaceutical Co., Tokyo, Japan) supplemented with $0.15 \%$ $\mathrm{NaHCO}_{3}, 10 \%$ bovine calf serum (JRH Biosciences, U.S.A.), penicillin (10 units $/ \mathrm{ml})$ and streptomycin $(10 \mathrm{mg} / \mathrm{ml})$. CHO cells (cell line derived from Chinese hamster ovarian carcinoma) for attached culture were grown in HAM's F12 medium (Nissui). CHO cells in suspension culture were grown in a serum-free CHO-S-SFM II medium (Invitrogen, Carlsbad, California, U.S.A.) with $0.15 \% \mathrm{NaHCO}_{3}$ for the production of recombinant proteins. To adapt $\mathrm{CHO}$ cells from the serum-supplemented medium to the serum-free medium, sequential adaptation was performed according to the instructions of the manufacturer (Invitrogen). The UV 92 cell line (mouse vascular endothelial cell line transformed by ultraviolet radiation) was purchased from RIKEN BioResourse Center (Tsukuba, Japan). UV $q 2$ cells were grown in DMEM with $0.15 \% \mathrm{NaHCO}_{3}$ and $10 \%$ fetal bovine serum (FBS) according to the provided protocol.

Plasmid Construction The following expression plasmids were constructed. pSec-F-sTNX (Fig. 1C), which encodes $200-\mathrm{kDa}$ sTNX with 15 FNIII repeats and a fibrinogen domain identical to the C-terminal portion of $450-\mathrm{kDa}$ iTNX, was constructed as follows. Spleens from adult ICR mice were dissected, frozen, and powdered in liquid nitrogen. Total RNA was isolated, and first-strand cDNA was synthesized by reverse transcription-polymerase chain reaction (RTPCR) as described previously. ${ }^{28)}$ First, pT7-M16j-19 was prepared by PCR using the prepared cDNA as a template and forward Eco-fM16-17 junction primer 5'-GGGAATTCGCTGTTGGGGTGACAGCTGCA-3' and reverse rM19 primer 5'-GGCTCGAGCACACCCACAGTCGACACGGG-3'. The PCR product was cloned into pT7Blue vector (Novagen, Madison, Wisconsin, U.S.A.), yielding pT7-M16j-19, which encodes the 16th-19th FNIII repeats. PCR was carried out according to the method described in our previous paper. ${ }^{28)}$ Next, pGEM-M19-25 was prepared by PCR using the prepared cDNA as a template and forward fM19 primer 5'GGGAATTCAGAGCCCCCGAGCCCAGAG-3' and reverse
del-rM25 primer 5'-GAGCCGTGGTGCCTTCCACGGAGAC-3'. PCR fragment was cloned into pGEM-T Easy vector (Promega, South San Francisco, CA, U.S.A.), yielding pGEM-M19-25, which encodes the 19th-25th FNIII repeats. Then a NaeI site in the vector sequence of pGEMM19-25 was changed to HindIII site by HindIII linker ligation. Subsequently, the EcoRI-SalI fragment of pT7-M16j19 and the SalI-HindIII fragment of pGEM-M19-25 were ligated and then inserted into the respective sites of pGEM-T Easy vector, in which the NaeI site in the vector sequence was changed to HindIII site, yielding pGEM-M16j-25. Finally, the EcoRI-ApaLI fragment of pGEM-M16j-25 and the ApaLI-XhoI fragment of the plasmid pB3CHX-18 encoding the 13 th -15 th and 25 th -31 st FNIII repeats followed by a fibrinogen domain were ligated and then inserted into the respective sites of $\mathrm{pSec}-\mathrm{F}$ vector, a $\mathrm{pSecTag} 2 / \mathrm{HygroB}$ vector (Invitrogen) containing a FLAG-tag, ${ }^{27}$ yielding FLAGtagged pSec-F-sTNX.

pSec-F-heptad-M16 encoding 250-kDa iTNX (Fig. 1B), which lacks the region of $200-\mathrm{kDa}$ sTNX from $450-\mathrm{kDa}$ iTNX, was constructed as follows. First, pGEM-M13-16 was constructed by PCR using the cDNA prepared from RNA from mouse spleen as a template and forward fM1314 primer 5'-GGGGATCCGACCCCAGAGAAACAACA$3^{\prime}$ and reverse rM16 primer 5'-GGCTCGAGAGACACAGGGCCCACACG-3'. The PCR product was cloned into pGEM-T Easy vector, yielding pGEM-M13-16, which encodes the 13 th -16 th FNIII repeats. Next, the HindIII-XhoI fragment of pGEM-M13-16 was inserted into HindIII/XhoI sites of pEGLNH-1 encoding the 10th-18th EGF repeats and the $1 \mathrm{st}-2$ nd and 4 th-13th FNIII repeats, yielding pBlue-EGF10-M16. Finally, the NotI-XhoI fragment of pBlue-EGF10-M16 was inserted into NotI/XhoI sites of pSecF2-1.1EGF-1 encoding a cysteine-rich segment at the amino terminus and the 1 st - 10th EGF repeats, yielding FLAG-tagged pSec-F-heptad-M16.

pSec-F-TNX, which encodes the full-length $450-\mathrm{kDa}$ iTNX (Fig. 1A), was constructed as follows. First, pGEMM13-17 was constructed by PCR using the cDNA prepared from mouse spleen as a template and forward fM13-14 primer and reverse rM17 primer 5'-GGCTCGAGCACACGACGCCCCTCATGGAG-3'. The PCR product was cloned into pGEM-T Easy vector, yielding pGEM-M13-17, which encodes the 13 th -17 th FNIII repeats. Then the $B \ln \mathrm{I}-X h o \mathrm{I}$ fragment of pGEM-M13-17 was replaced by the $B \ln \mathrm{I}-X h o \mathrm{I}$ fragment of pSec-F-sTNX, yielding pGEM-M13-FG. Next, the HindIII-XhoI fragment of pGEM-M13-FG was inserted into the HindIII/XhoI sites of pEGLNH-1, yielding pBlueEGF10-FG. Finally, the NotI-XhoI fragment of pBlueEGF10-FG was inserted into NotI/XhoI sites of pSecF21.1EGF-1, yielding FLAG-tagged pSec-F-TNX.

pSecFTNX-2 encoding a FLAG-tagged splice isoform of 340-kDa iTNX, which lacks M3 and M15-M22 fibronectin type III repeats, pSec-mVA ${ }_{164}-\mathrm{HA}$ encoding HA-tagged mouse VEGF-A ${ }_{164}$, and pSec-hVB ${ }_{186}$-HA encoding HAtagged human VEGF-B ${ }_{186}$ have been described previously. ${ }^{27)}$

Establishment of 200-kDa sTNX, 250-kDa iTNX and 450-kDa iTNX-Expressing Cell Lines Adherent CHO cells in a $10-\mathrm{cm}$ dish were transfected with $5 \mu \mathrm{g}$ of pSec-FsTNX, pSec-F-heptad-M16 or pSec-F-TNX by the calcium phosphate precipitation technique ${ }^{29)}$ and cultured in the pres- 
ence of $800 \mu \mathrm{g} / \mathrm{ml}$ of hygromycin B (Wako, Osaka, Japan). About 2 weeks after transfection, hygromycin B-resistant colonies were selected and used as 200-kDa sTNX, 250-kDa iTNX or 450-kDa iTNX-expressing cell lines. As a control, mock cells carrying an empty $\mathrm{pSecTag} 2 / \mathrm{HygroB}$ vector were also selected.

Western Blot Proteins in cell lysates or conditioned medium were electrophoresed through $7.5 \%$ or $10 \%$ sodium dodecyl sulfate (SDS)-polyacrylamide gels and then electroblotted (PAGE) onto Hybond ECL nitrocellulose membranes (GE Healthcare, Buckinghamshire, England). The membranes were blocked at room temperature (RT) for $1 \mathrm{~h}$ with 5\% nonfat dry milk in TBST $[20 \mathrm{~mm}$ Tris- $\mathrm{HCl}(\mathrm{pH}$ 7.5), $150 \mathrm{~mm} \mathrm{NaCl}, 0.1 \%$ Tween-20] and then incubated with a primary antibody $(10 \mu \mathrm{g} / \mathrm{ml}$ in TBST with $1 \%$ milk) for $1 \mathrm{~h}$ at RT. Membranes were washed three times with TBST, incubated with anti-mouse, anti-rabbit, anti-goat Alexa Flour 680-conjugated or IRDye 800-conjugated immunoglobulin (Ig)G (Molecular Probes, Eugene, OR, U.S.A.) followed by visualization using an infrared imaging system (Odyssey, LICOR, Lincoln, U.S.A.).

Detection of 200-kDa sTNX, 250-kDa iTNX or 450-kDa iTNX in Conditioned Medium, Compared with That in Cell Lysates For the detection of FLAG-tagged 200-kDa sTNX, $250-\mathrm{kDa}$ iTNX or $450-\mathrm{kDa}$ iTNX in the conditioned medium of the cell line expressing each type of TNX cultivated for $48 \mathrm{~h}$, the conditioned medium was clarified from debris by centrifugation. Subsequently, anti-FLAG M2 affinity resin (Sigma-Aldrich, Tokyo, Japan) equilibrated in phosphate-buffered saline (PBS) $/ 0.5 \%$ NP-40 was added to the collected medium, followed by rotation at $4{ }^{\circ} \mathrm{C}$ for $3 \mathrm{~h}$. The resin was pelleted by centrifugation and washed several times with wash buffer $[10 \mathrm{~mm}$ Tris- $\mathrm{HCl}(\mathrm{pH} 7.5), 50 \mathrm{~mm}$ $\mathrm{NaCl}, 0.1 \%$ Nonidet P-40 (NP-40)]. Afterwards, the resin was boiled in Laemmli buffer before being subjected to SDS/PAGE and Western blot analysis with anti-FLAG M2 monoclonal antibody (Sigma-Aldrich). On the other hand, for the detection of each TNX form in cells, cells were lysed in lysis buffer containing $50 \mathrm{~mm}$ Tris- $\mathrm{HCl}$ (pH 7.5), $120 \mathrm{~mm}$ $\mathrm{NaCl}, 0.5 \% \mathrm{NP}-40$, and protease inhibitors for $10 \mathrm{~min}$ on ice, and whole-cell lysates were obtained by subsequent centrifugation. The protein concentration was determined by a bicinchoninic acid (BCA) assay (Pierce, Rockford, U.S.A.). Cell lysates were electrophoresed and subjected to Western blot analysis.

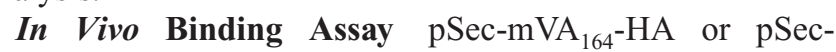
$\mathrm{hVB}_{186}$-HA was transfected into $293 \mathrm{~T}$ cells with each FLAGtagged TNX form (200-kDa sTNX, 250-kDa iTNX and 450$\mathrm{kDa}$ iTNX), and then $48 \mathrm{~h}$ after transfection the cell lysate was prepared as described in the above section. Approximately $3 \mathrm{mg}$ of $293 \mathrm{~T}$ cell proteins was first immunoprecipitated with mouse anti-FLAG M2-agarose affinity resin at $4{ }^{\circ} \mathrm{C}$ for $4 \mathrm{~h}$. After centrifugation and washing of the resin five times with wash buffer, the precipitates were separated in a $7.5 \%$ SDS-polyacrylamide gel and electroblotted onto nitrocellulose filter membranes. Then the membranes were incubated with a specific antibody against HA (Bethyl Laboratories, Montgomery, TX, U.S.A.). After washing, the membranes were incubated with Alexa Fluor 680-conjugated antirabbit or IRDye800-conjugated anti-mouse antibodies followed by visualization using infrared imaging.
Purification of Recombinant 200-kDa sTNX from Conditioned Medium To cultivate adherent 200-kDa sTNXexpressing cell lines in suspension culture, sequential adaptation of the 200-kDa sTNX-expressing cell line from serumsupplemented medium to serum-free CHO-S-SFM II medium was performed. To isolate the recombinant $200-\mathrm{kDa}$ sTNX from the conditioned medium of sTNX-expressing cells in suspension culture, approximately 11 of the conditioned medium was collected and centrifuged to remove cell debris. Subsequently, the cleared conditioned medium was precipitated with $50 \%$ ammonium sulfate by stirring overnight at $4{ }^{\circ} \mathrm{C}$. After centrifugation for $20 \mathrm{~min}$ at $12000 \mathrm{rpm}$, the pellet was dissolved in $10 \mathrm{ml}$ of high-salt buffer containing $0.5 \mathrm{M} \mathrm{NaCl}$ and $20 \mathrm{~mm}$ Tris- $\mathrm{HCl}$ (pH 7.5) and dialyzed overnight against the same buffer. Sepharose 4B resin (Sigma-Aldrich) coupled with the anti-mouse tenascin$\mathrm{X}$ antibody pAb10d211 ${ }^{20)}$ was resuspended in the dialyzed sample and incubated overnight at $4{ }^{\circ} \mathrm{C}$. The resin was then washed extensively in the high-salt buffer. The antigen was eluted with $200 \mathrm{~mm}$ triethylamine in the high-salt buffer, and the eluate was immediately neutralized with $2 \mathrm{M}$ Tris- $\mathrm{HCl}$ (pH 7.0). The eluate containing sTNX was analyzed by $7.5 \%$ SDS-PAGE under reducing conditions and visualized by silver staining (Wako). Approximately $6 \mu \mathrm{g}$ of the recombinant sTNX was obtained from 2 liters of the conditioned medium. As a negative control, the proteins were purified from conditioned medium of mock cells that carry an empty expression vector.

Cell Proliferation Assay by 5-Ethynyl-2'-deoxyuridine (EdU) Labeling UV 92 cells were grown overnight in 8well culture slides (BD Biosciences, San Jose, CA, U.S.A.) in DMEM supplemented with $10 \%$ fetal bovine serum (FBS), penicillin, and streptomycin. Then the conditioned medium was changed to fresh medium containing $0.5 \% \mathrm{FBS}$ and VEGF $_{165}\left(V_{E G F-A}{ }_{165}\right)(25 \mathrm{ng} / \mathrm{ml})$ (PeproTech, Rocky Hill, NJ, U.S.A.), bFGF (25 ng/ml) (PeproTech) and/or recombinant $200-\mathrm{kDa}$ sTNX $(10,50,100 \mathrm{ng} / \mathrm{ml})$. As a negative control, conditioned medium from mock cells was added. EdU labeling of proliferative cells was done according to the instruction manual for Click-iT ${ }^{\mathrm{TM}}$ EdU Alexa Fluor HighThrough Put Imaging (HCS) Assay (Invitrogen). After $32 \mathrm{~h}$, EdU was added to the culture medium at the concentration of $10 \mu \mathrm{M}$ for $10 \mathrm{~h}$. After labeling, cells were washed three times with phosphate-buffered saline (PBS) and were fixed with $4 \%$ paraformaldehyde for $15 \mathrm{~min}$. After rinsing with $3 \%$ bovine serum albumin (BSA) in PBS, cells were permeabilized with $0.5 \%$ Triton X-100 in PBS for 20 min. Then cells were stained for $30 \mathrm{~min}$ with Click-iT ${ }^{\mathrm{TM}}$ reaction cocktails (Invitrogen). Nuclear DNA was visualized with 4',6-diamidino-2-phenylindole (DAPI) $(1 \mu \mathrm{g} / \mathrm{ml})$. The cells were then observed using an All-In-One (BZ-8000) fluorescent microscopy (Keyence, Osaka, Japan). Then stained cells were counted.

\section{RESULTS}

Easier Release of sTNX into Conditioned Medium than That of 450-kDa iTNX and 250-kDa iTNX As shown in our previous study, ${ }^{20)} 200-\mathrm{kDa}$ sTNX is abundantly present in serum. Therefore, we speculated that $200-\mathrm{kDa}$ sTNX is more easily released than $450-\mathrm{kDa}$ iTNX from cells into ex- 
tracellular space. In order to examine how $200-\mathrm{kDa}$ sTNX is easily released to extracellular space, at first we attempted to construct an expression plasmid, pSec-F-sTNX, encoding FLAG-tagged 200-kDa sTNX as described in Materials and Methods. As a control, we also constructed an expression plasmid encoding FLAG-tagged 450-kDa iTNX and an expression plasmid encoding FLAG-tagged 250-kDa iTNX, which lacks the region of $200-\mathrm{kDa}$ sTNX from $450-\mathrm{kDa}$ iTNX (Figs. 1A, B, C). We transfected these plasmids into CHO cells and established stable cell lines expressing 200kDa sTNX, 450-kDa iTNX and 250-kDa iTNX. To confirm the expression of each TNX in the stable cell lines, cell lysates were prepared and immunoblot analyses of each TNX were performed with the use of two polyclonal antibodies, pAbM11 and pAb10d211, specific for different FNIII repeats of mouse TNX. Since the pAbM11 and pAb10d211 antibodies recognize the 11th FNIII repeat (M11) and the 25th FNIII to the 30th FNIII repeat (M25-M30) of mouse TNX, respectively (Fig. 1D), ${ }^{20)}$ it was expected that the pAbM11 $(\alpha-$ M11) antibody would detect $250-\mathrm{kDa}$ iTNX and $450-\mathrm{kDa}$ iTNX, while the pAb10d211 ( $\alpha$-10d211) antibody would detect $200-\mathrm{kDa}$ sTNX and 450-kDa iTNX. As expected, 200-

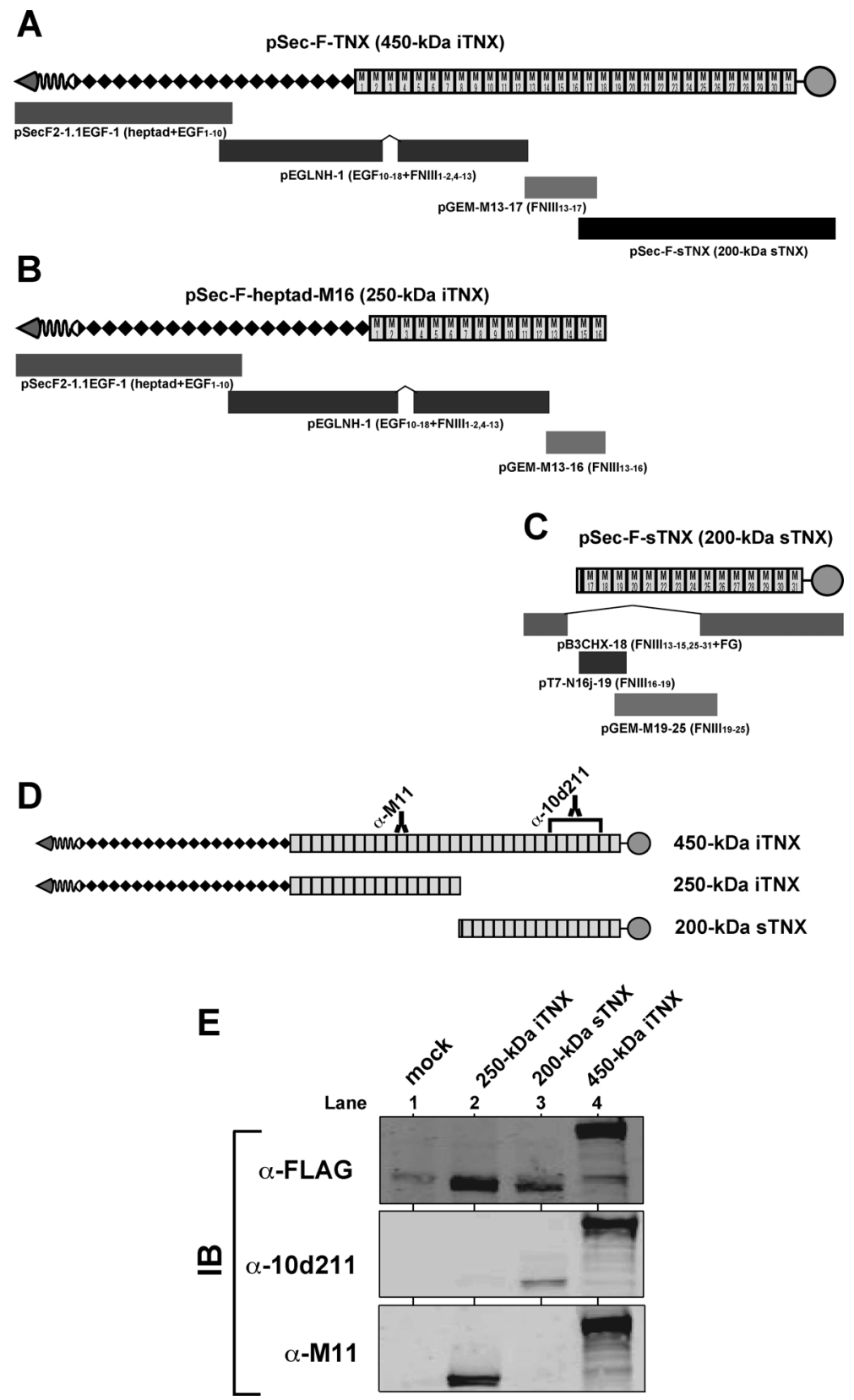

Fig. 1. Establishment of 200-kDa sTNX, 250-kDa iTNX and 450-kDa iTNX-Expressing Cell Lines

Schematic diagrams of the domain structure of each recombinant TNX, recombinant 450-kDa iTNX (A), 250-kDa iTNX (B), and 200-kDa sTNX (C). The recombinant protein is depicted as a linear array from the N-terminus to C-terminus showing the central domain (segment of circle), heptad repeats (wavy line), 18.5 EGF-like repeats (diamonds), 31 FNIII-like repeats (M1-M31) (rectangles) and the fibrinogen-like domain (circle). The horizontal boxes under the diagrams of each recombinant TNX show the encompassed regions of the plasmid DNA clones used for the construction of the recombinant TNX with its designation of each plasmid. The encompassed domain region of each plasmid used is indicated in parenthesis. $\wedge$ indicates contiguity. (D) FNIII domains in which pAbM11 ( $\alpha$-M11) and pAb10d211 ( $\alpha$-10d211) antibodies are able to recognize each recombinant TNX. The pAbM11 antibody recognizes the 11th FNIII (M11) repeat, whereas the pAb10d211 antibody recognizes the 25th to the 30th FNIII repeats of mouse TNX. (E) Expression of 200-kDa sTNX, 250-kDa iTNX and 450-kDa iTNX in corresponding cell lines. Cell lysates from mock cells (lane 1), 250-kDa iTNX-expressing cells (lane 2), 200-kDa sTNX-expressing cells (lane 3), and 450-kDa iTNX-expressing cells (lane 4) cell lines were immunoblotted with anti-FLAG ( $\alpha$-FLAG), pAbM11 ( $\alpha$-M11), or pAb10d211 ( $\alpha$ $10 \mathrm{~d} 211)$ antibody. 
A

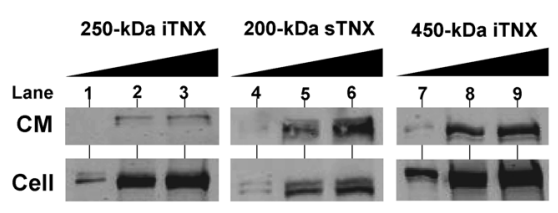

IB: $\alpha$-FLAG

B

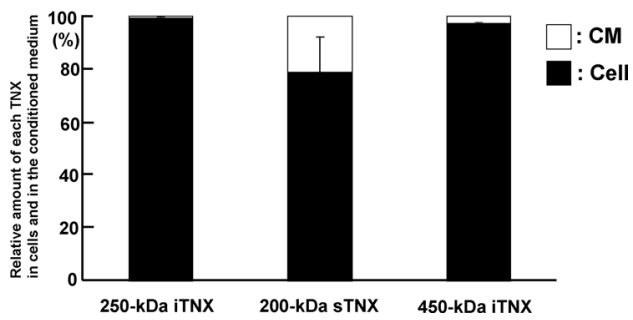

Fig. 2. The 200-kDa sTNX Is Easily Released into the Conditioned Medium Compared with 250-kDa iTNX and 450-kDa iTNX

(A) Western blot analysis to determine the amounts of 200-kDa sTNX, 250-kDa iTNX and 450-kDa iTNX released into the conditioned medium. (Lower panel, Cell) Various amounts of cell lysates from 250-kDa iTNX (lanes 1-3), 200-kDa sTNX (lanes 4 -6), and 450-kDa iTNX (lanes 7-9) were immunoblotted with anti-FLAG antibody. Ten (lanes 1, 4, 7), fifty (lanes 2, 5, 8), and seventy-five (lanes 3, 6, 9) micrograms of cell lysates from each cell line were applied to SDS/PAGE. (Upper panel, $\mathrm{CM})$ The conditioned medium corresponding to the amount of each cell lysate was immunoprecipitated with anti-FLAG M2 monoclonal antibody and the precipitates were blotted with the anti-FLAG antibody. (B) Quantification of the percentage of the released TNX into the conditioned medium versus TNX in the cell lysate for $250-\mathrm{kD}$ iTNX, 200-kDa sTNX and 450-kDa iTNX-expressing cell lines. Bands in (A) were scanned with an Odyssey apparatus, and their relative intensities are represented by histograms.

kDa sTNX was only detected by the pAb10d211 antibody, whereas $250-\mathrm{kDa}$ iTNX was only detected by the pAbM11 antibody (Fig. 1E). Furthermore, 450-kDa iTNX was detected by both antibodies, and the anti-FLAG antibody detected all of three recombinant TNX forms. However, neither of these antibodies detected the proteins from lysates of mock cells carrying an empty pSecTag2/HygroB vector. These results indicated that the cell lines bearing the 200kDa sTNX, 250-kDa iTNX and 450-kDa iTNX recombinant plasmids, express the corresponding proteins precisely.

Next, in order to compare the amounts of 200-kDa sTNX, 250-kDa iTNX and 450-kDa iTNX released into conditioned medium from these established cell lines for $48 \mathrm{~h}$, we detected each TNX both in cell lysates that have a certain fixed amount of total proteins and in its corresponding conditioned medium by Western blot analyses (Fig. 2A). Subsequently, we measured the intensity of the band derived from each TNX both in cells and in the corresponding conditioned medium, and we calculated the ratio of each released TNX in the conditioned medium to the total quantity of each expressed TNX (Fig. 2B). The ratios of TNX released into the conditioned medium to total expressed TNX were approximately $20.8 \%, 2.7 \%$ and $1.0 \%$ for $200-\mathrm{kDa}$ sTNX, $450-\mathrm{kDa}$ iTNX and $250-\mathrm{kDa}$ iTNX, respectively. These results indicated that $200-\mathrm{kDa}$ sTNX is more easily released than 250 $\mathrm{kDa}$ iTNX and 450-kDa iTNX into the conditioned medium.

200-kDa sTNX Strongly Binds to VEGF-A and VEGFB We have reported that a splice isoform of $340-\mathrm{kDa}$ iTNX that lacks M3 and M15-M22 FNIII repeats binds to VEGF$\mathrm{A}$ and VEGF-B. ${ }^{27}$ ) Since we were interested in knowing whether $200-\mathrm{kDa}$ sTNX also interacts with VEGF-A and
A
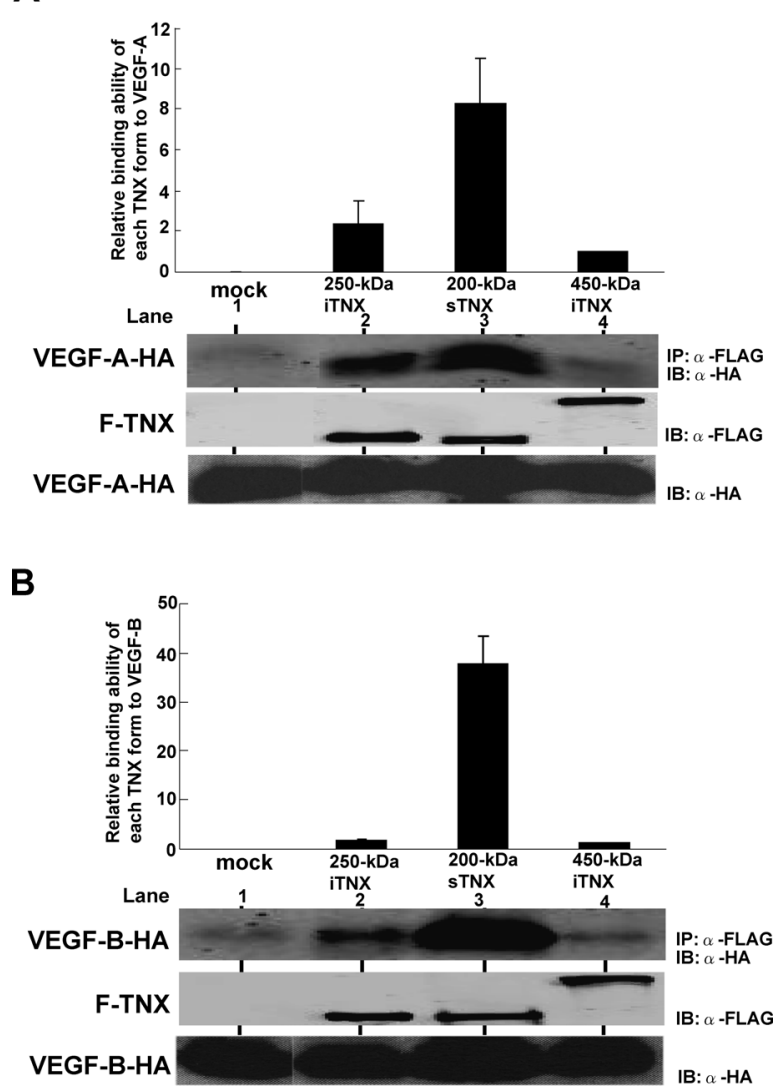

Fig. 3. Strong Binding of 200-kDa sTNX to VEGF-A and VEGF-B

Binding of each TNX to VEGF-A (A) and VEGF-B (B). 293T cells were transfected with expression vectors for FLAG-tagged 250-kDa iTNX (lane 2), 200-kDa sTNX (lane 3), and 450-kDa iTNX (lane 4) in combination with HA-tagged VEGF-A (A) or VEGF-B (B) by the calcium phosphate precipitation technique, and the cell extract was prepared $48 \mathrm{~h}$ after transfection. Proteins in the extract were first precipitated with an anti-FLAG antibody, and the precipitates were immunoblotted with an anti-HA antibody [upper panel, VEGF-A-HA (A) or VEGF-B-HA (B)]. To confirm the expression of each TNX, 1/100 amount of the proteins (input) used for the immunoprecipitation reactions was run in parallel and immunoblotted with an anti-FLAG antibody [middle panel, F-TNX (A and B)]. To confirm equivalent levels of the expression of HA-tagged VEGF-A (A) and VEGF-B (B), 1/50 amount of the proteins (input) used for the immunoprecipitation reactions was run in parallel and immunoblotted with an anti-HA antibody [lower panel, VEGF-A-HA (A) or VEGF-B-HA (B)]. IP, immunoprecipitation: IB, immunoblot. Relative binding abilities of each TNX to VEGF-A (A) and VEGF-B (B) are also shown. The band intensity of $450-\mathrm{kDa}$ iTNX [lane 4 in upper panels in both $(\mathrm{A})$ and $(\mathrm{B})$ ] was considered as 1.0 .

VEGF-B, the ability of VEGF-A and VEGF-B to bind to 200-kDa sTNX was investigated by a co-immunoprecipitation assay. As a comparison, the abilities of $250-\mathrm{kDa}$ iTNX and $450-\mathrm{kDa}$ iTNX to bind to VEGF-A and VEGF-B were also investigated. To observe the interaction of each form of TNX with VEGF-A and VEGF-B in vivo, expression vectors for each FLAG-tagged form of TNX and HA-tagged VEGFA or HA-tagged VEGF-B were together transfected into human 293T cells. Forty-eight hours after transfection, the cell extract was prepared and the proteins in the extract were first immunoprecipitated with the anti-FLAG antibody. The precipitates were immunoblotted against the anti-HA antibody. The anti-FLAG antibody precipitated each FLAGtagged form of TNX. On the other hand, HA-tagged VEGFA (Fig. 3A) or VEGF-B (Fig. 3B) was detected in the immunoprecipitates with the anti-FLAG antibody. The equivalent levels of the expression of HA-tagged VEGFs were confirmed by immunoblot analysis with anti-HA antibody of the 
cell extracts (Fig. 3). The relative binding ability of each TNX form to VEGF-A or VEGF-B was compared by measuring the intensity of each band. It was found that the abilities of 200-kDa sTNX to VEGF-A and VEGF-B were approximately 8 -fold and 38-fold greater, respectively, than those of $450-\mathrm{kDa}$ iTNX. These results indicate that $200-\mathrm{kDa}$ sTNX strongly binds to VEGF-A and VEGF-B, compared to the binding abilities of $250-\mathrm{kDa}$ iTNX and 450-kDa iTNX.

200-kDa sTNX Possesses Weak Activity for Proliferation of Endothelial Cells We previously showed that 340$\mathrm{kDa}$ iTNX alone has weak activity for proliferation of endothelial cells, whereas $340-\mathrm{kDa}$ iTNX in combination with VEGF-B ${ }_{186}$ strongly promotes endothelial cell proliferation compared with the effect of VEGF-B ${ }_{186}$ alone. ${ }^{27)}$

It is known that VEGF-A and basic fibroblast growth factor (bFGF), also known as FGF-2, stimulate endothelial cell proliferation. $^{30)}$ To investigate the effect of purified $200-\mathrm{kDa}$ sTNX (Fig. 4A) alone and in combination with VEGF-A 165 and $\mathrm{bFGF}$ on endothelial cell proliferation, UV $\$ 2$ endothelial cells were stimulated by purified recombinant $200-\mathrm{kDa}$ sTNX alone and in combination with VEGF-A $\mathrm{A}_{165}$ and bFGF. Then cell proliferation was examined by analysis of EdU incorporation in UV $q 2$ cells. As shown in Fig. 4B, VEGF-A 165 increased EdU incorporation into DNA of UV +2 cells (1.6fold induction of that of non-treated cells). Interestingly, 200kDa sTNX alone also stimulated endothelial cell proliferation to an extent similar to that induced by VEGF-A ${ }_{165}(1.4-$ fold induction of that of non-treated cells at $100 \mathrm{ng} / \mathrm{ml}$ ). Furthermore, 200-kDa sTNX together with VEGF-A $\mathrm{A}_{165}$ increased EdU incorporation into DNA by 1.9 fold compared with that of non-treated cells. These results demonstrate that the effect of purified 200-kDa sTNX with VEGF-A 165 on endothelial cell proliferation is greater than that of the $200-\mathrm{kDa}$ sTNX alone.

We also examined the effect of 200-kDa sTNX together with bFGF on endothelial cell proliferation. As shown in Fig. $4 \mathrm{C}$, bFGF increased EdU incorporation to 1.5 fold compared with that of non-treated cells. Moreover, 200-kDa sTNX together with bFGF increased the 2.0-fold incorporation compared with that of non-treated cells, demonstrating again that the effect of purified 200-kDa sTNX with bFGF on endothelial cell proliferation is greater than that of $200-\mathrm{kDa}$ sTNX alone. These results indicate that $200-\mathrm{kDa}$ sTNX alone has weak activity for endothelial cell proliferation and that 200kDa sTNX in combination with growth factors such as VEGF-A ${ }_{165}$ and bFGF shows slightly stronger endothelial cell proliferation activity than that of $200-\mathrm{kDa}$ sTNX alone.

\section{DISCUSSION}

This is the first report on characterization of $200-\mathrm{kDa}$ sTNX. The amount of $200-\mathrm{kDa}$ sTNX released into conditioned medium is 7-fold larger than that of $250-\mathrm{kDa}$ iTNX or 450-kDa iTNX. In addition, sTNX strongly binds to VEGF$A$ and VEGF-B compared with the binding abilities of other iTNX proteins. The purified $200-\mathrm{kDa}$ sTNX both alone and in combination with VEGF-A or bFGF possesses weak activity for endothelial cell proliferation.

The binding sites of mouse $450-\mathrm{kDa}$ iTNX to cells have not yet been determined. However, for bovine TNX it has been reported that the contiguous $\mathrm{b} 9$ and b10 FNIII repeats
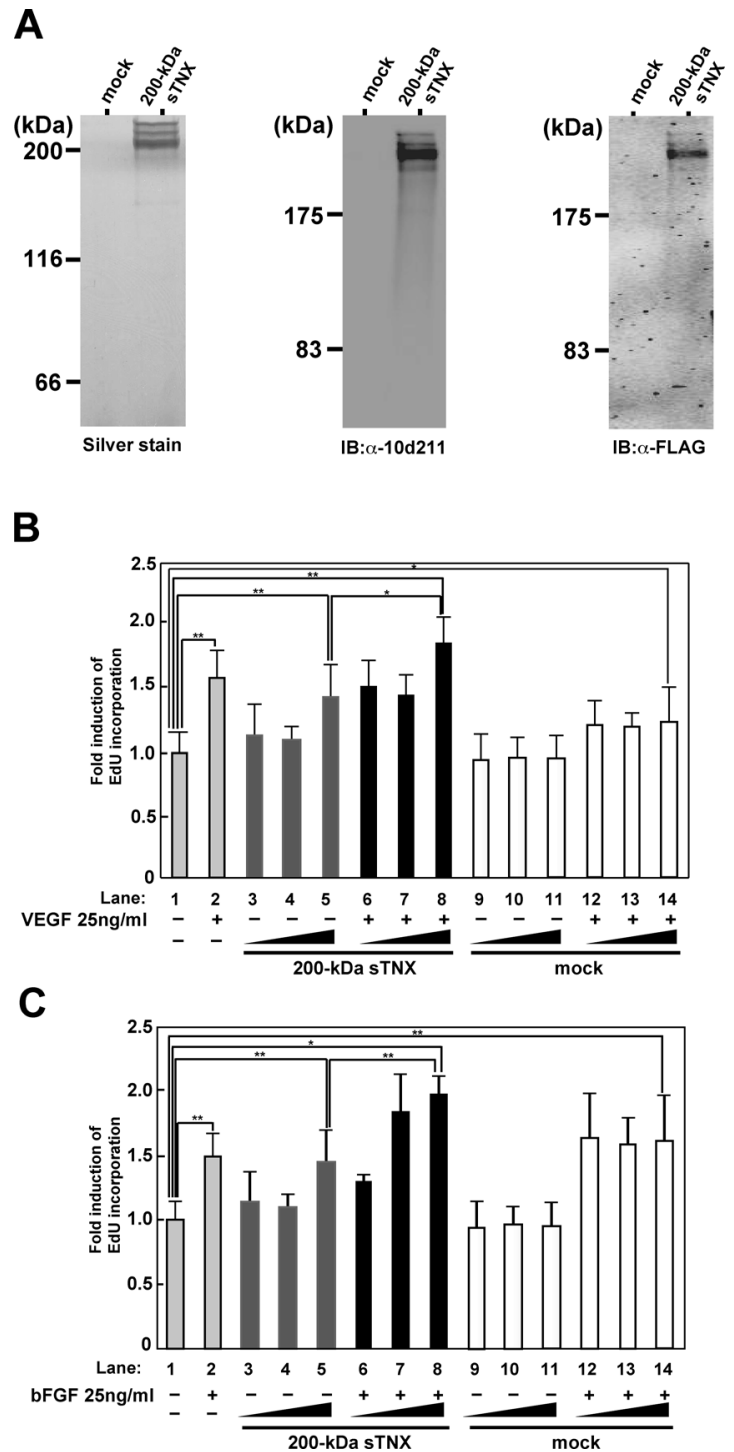

Fig. 4. Weak Endothelial Cell Proliferation Activity of Purified 200-kDa sTNX in Combination with VEGF-A or bFGF

(A) Purification of the recombinant 200-kDa sTNX from conditioned medium. (Left panel) Immunopurification of 200-kDa sTNX from the conditioned medium of 200$\mathrm{kDa}$ sTNX-expressing CHO cells in suspension culture. The recombinant 200-kDa sTNX was immunopurified using the TNX-specific antibody pAb10d211. A representative silver stained gel is shown. As a negative control, the proteins were purified from conditioned medium of mock cells. (Middle panel) Immunoblot analysis of the purified recombinant 200-kDa sTNX from the conditioned medium using pAb10d211 antibody. (Right panel) Immunoblot analysis of the purified 200-kDa sTNX from the conditioned medium using anti-FLAG antibody. In (B), $25 \mathrm{ng} / \mathrm{ml}$ VEGF-A (lane 2), various concentrations of sTNX (lane $3,10 \mathrm{ng} / \mathrm{ml}$; lane $4,50 \mathrm{ng} / \mathrm{ml}$; lane $5,100 \mathrm{ng} / \mathrm{ml}$ ) alone, various concentrations of sTNX (lane $6,10 \mathrm{ng} / \mathrm{ml}$; lane $7,50 \mathrm{ng} / \mathrm{ml}$; lane $8,100 \mathrm{ng} / \mathrm{ml}$ ) with $25 \mathrm{ng} / \mathrm{ml}$ VEGF-A, various concentrations of proteins purified from conditioned medium of mock cells (lane $9,10 \mathrm{ng} / \mathrm{ml}$; lane $10,50 \mathrm{ng} / \mathrm{ml}$; lane $11,100 \mathrm{ng} / \mathrm{ml}$ ) alone, or various concentrations of proteins purified from conditioned medium of mock cells (lane $12,10 \mathrm{ng} / \mathrm{ml}$; lane $13,50 \mathrm{ng} / \mathrm{ml}$; lane $14,100 \mathrm{ng} / \mathrm{ml}$ ) with $25 \mathrm{ng} / \mathrm{ml}$ VEGF-A was applied to UV +2 endothelial cells, and incorporation of EdU was measured. The columns show fold induction of EdU incorporation compared to basal activity induced by the control medium only (lane 1 ). In (C), $25 \mathrm{ng} / \mathrm{ml} \mathrm{bFGF}$ was used in place of VEGF-A in (B). The data in lanes 1, 3, 4, 5, 9, 10 and 11 in (B) and those in lanes 1, 3, $4,5,9,10$ and 11 in $(\mathrm{C})$ are the same data, respectively. $* * p<0.01$ and $* p<0.05$, Mann-Whitney $U$ test; statistically significant.

and the fibrinogen domain participate in cell adhesion via $\alpha v \beta 3$ integrin and $\beta 1$-containing integrin receptors, respectively. $^{31)}$ Furthermore, the same group ${ }^{32}$ identified two FNIII repeats, b10 and b11, of bovine TNX in which heparin-binding sites exist. They also showed that the b10 and b11 repeats are involved in cell adhesion mediated by heparan sulfate cell 
surface receptors. We previously performed phylogenetic analysis for 94 FNIII repeats derived from human, mouse and bovine $\mathrm{TNX}^{28)}$ The results of this analysis showed that the b9, b10 and b11 FNIII repeats of bovine TNX have high similarity to the 10th (M10), 11th (M11) and 12th (M12) FNIII repeats in the 31 FNIII repeats of mouse TNX, respectively. Therefore, it is possible that mouse M11 and M12 FNIII repeats of $250-\mathrm{kDa}$ iTNX and $450-\mathrm{kDa}$ iTNX possess heparin-binding activity. Since heparan sulfate/heparin (e.g., heparan sulfate cell surface receptors) is prominently present on the cell membrane, $250-\mathrm{kDa}$ iTNX and 450-kDa iTNX with M11 and M12 repeats might interact with cell membranes and adhere to cells. Actually, in this study (Fig. 2), we showed that 200-kDa sTNX, which lacks the 250-kDa N-terminal region (including the M11 and M12 FNIII repeats) of $450-\mathrm{kDa}$ iTNX, is released into conditioned medium and is not easily maintained on the cell surface compared with 250kDa iTNX and 450-kDa iTNX.

200-kDa sTNX strongly bound to VEGFs compared with the binding abilities of $250-\mathrm{kDa}$ iTNX and $400-\mathrm{kDa}$ iTNX. We previously examined the interaction sites of $340-\mathrm{kDa}$ iTNX with VEGF-B and found that the mTNX/FNIII ${ }_{13-25}$ region (encoding M13, M14, M23, M24 and M25 FNIII repeats) of $340-\mathrm{kDa}$ iTNX interacts with VEGF-B. ${ }^{27)}$ Thus, judging from these previous experimental findings and the present results, we predict that the interaction sites of 200kDa sTNX having M17-M31 FNIII repeats and a fibrinogen-like domain with VEGF-B might be localized within M23, M24 and M25 FNIII repeats. Although 250-kDa iTNX does not include the M23, M24 and M25 repeats, it binds to VEGF-B weakly compared with $200-\mathrm{kDa}$ sTNX. This might be due to the fact that $250-\mathrm{kDa}$ iTNX has the 10th (M10) and 15th (M15) FNIII repeats with high similarity to the M23, $\mathrm{M} 24$ and M25 repeats. ${ }^{28)}$ On the other hand, for the reason why $450-\mathrm{kDa}$ iTNX having M23, M24 and M25 repeats binds to VEGFs very faintly, we speculated that the M23, M24 and M25 repeats are masked by N-terminal regions of 450-kDa iTNX which 200-kDa sTNX lacks and consequently VEGFs cannot approach the M23, M24 and M25 repeats of 450-kDa iTNX.

The 200-kDa sTNX alone displays weak endothelial cell proliferation activity (Fig. 4). The reason for this is not clear at present. This proliferation might be mediated by unidentified receptor(s) for $200-\mathrm{kDa}$ sTNX. However, it is also possible that $200-\mathrm{kDa}$ sTNX directly interacts with VEGF receptor (VEGFR-1 or VEGFR-2) and transmits signals from the receptor to regulate gene expression, leading to endothelial cell proliferation. We performed preliminary experiments on the interaction of $200-\mathrm{kDa}$ sTNX with VEGFR-1, and we found that the sTNX interacts with VEGFR-1 (data not shown). However, it has not been determined whether the interaction results in activation of VEGFR-1 and transmits signals to its downstream targets.

Previously, we ${ }^{27)}$ showed that $340-\mathrm{kDa}$ iTNX, a small splice isoform that lacks M3 and M15-M22 FNIII repeats of $450-\mathrm{kDa}$ iTNX, in combination with VEGF-B increased endothelial cell proliferation by 3.9 fold compared with that induced by VEGF-B alone. On the other hand, the present study demonstrated that 200-kDa sTNX in combination with VEGF-A increased endothelial cell proliferation by only 1.2 fold compared with that induced by VEGF-A alone. The major reason why 340-kDa iTNX with VEGF-B possesses a high level of endothelial cell proliferation activity, but 200kDa sTNX with VEGF-A has a little such activity, might be due to the difference of cell proliferation assay system and cells used between the previous study ${ }^{27}$ and this study. Namely, in previous study ${ }^{27}$ the cell proliferation was examined through an analysis of $\left[{ }^{3} \mathrm{H}\right]$ thymidine incorporation in bovine carotid artery endothelial $(\mathrm{HH})$ cells, and on the other hand in this study the cell proliferation was examined through 5-ethynyl-2'-deoxyuridine (EdU) incorporation in mouse endothelial UV +2 cells. Other conceivable reason might be as follows. Since 340-kDa iTNX has M11 and M12 FNIII repeats that bear possible heparin/heparan sulfatebinding sites, as in the case of $450-\mathrm{kDa}$ iTNX $340-\mathrm{kDa}$ iTNX might interact with heparan sulfate cell surface receptors and be maintained on cell membranes. The $340-\mathrm{kDa}$ iTNX associated with cell membranes might capture VEGF$\mathrm{B}$ close to the cell membrane and facilitate presentation of VEGF-B to its receptor VEGFR-1. On the other hand, as mentioned above, the rate of association of the $200-\mathrm{kDa}$ sTNX with the cell membrane is lower than that of the 340$\mathrm{kDa}$ iTNX and the sTNX is easily released into the extracellular environment. Due to the strong binding of VEGF-A and 200-kDa sTNX, the released sTNX might catch VEGF-A away from the cell membrane where VEGF-A receptors such as VEGFR-2 and VEGFR-1 are localized and transmit signals for cell proliferation. However, even though VEGF-A to some extent was trapped by the released sTNX in the extracellular environment, sTNX with VEGF-A would also stay on the cell membrane to some extent since $79.2 \%$ of 200 $\mathrm{kDa}$ sTNX exist in the cell and on the cell (Fig. 2B). Therefore, the left VEGF-A with sTNX associated with cell membranes might lead to the 1.2-fold induction of cell proliferation compared with that induced VEGF-A alone.

Further studies are needed to determine the endothelial cell proliferation activity of $450-\mathrm{kDa}$ iTNX alone, $200-\mathrm{kDa}$ sTNX with 450-kDa iTNX and also that of 200-kDa sTNX and $450-\mathrm{kDa}$ iTNX with VEGF-A or VEGF-B treated at the same time. It would be of great interest to investigate whether 200-kDa sTNX gives influence to the endothelial cell proliferation activity that $450-\mathrm{kDa}$ iTNX might possess with VEGF-A or VEGF-B.

Acknowledgments We thank Kiyomi Takaya for her technical assistance. This work was supported in part by Grants-in-Aid for Scientific Research from the Ministry of Education, Culture, Sports, Science and Technology of Japan.

\section{REFERENCES}

1) Adams J. C., Watt F. M., Development, 117, 1183-1198 (1993).

2) Chiquet-Ehrismann R., Mackie E. J., Pearson C. A., Sakakura T., Cell, 47, 131-139 (1986).

3) Fuss B., Wintergerst E. S., Bartsch U., Schachner M., J. Cell Biol., 120, 1237-1249 (1993).

4) Weber P., Montag D., Schachner M., Bernhardt R. R., J. Neurobiol., 35, 1-16 (1998).

5) Neidhardt J., Fehr S., Kutsche M., Lohler J., Schachner M., Mol. Cell Neurosci., 23, 193-209 (2003).

6) Scherberich A., Tucker R. P., Samandari E., Brown-Luedi M., Martin D., Chiquet-Ehrismann R., J. Cell Sci., 117, 571-581 (2004).

7) Burch G. H., Gong Y., Liu W., Dettman R. W., Curry C. J., Smith L., 
Miller W. L., Bristow J., Nat. Genet., 17, 104-108 (1997).

8) Schalkwijk J., Zweers M. C., Steijlen P. M., Dean W. B., Taylor G., van Vlijmen I. M., van Haren B., Miller W. L., Bristow J., N. Engl. J. Med., 345, 1167-1175 (2001).

9) Zweers M. C., Bristow J., Steijlen P. M., Dean W. B., Hamel B. C., Otero M., Kucharekova M., Boezeman J. B., Schalkwijk J., Am. J. Hum. Genet., 73, 214-217 (2003).

10) Wei J., Hemmings G. P., Am. J. Med. Genet. B. Neuropsychiatr. Genet., 125, 43-49 (2004).

11) Gardella R., Zoppi N., Assanelli D., Muiesan M. L., Barlati S., Colombi M., Am. J. Med. Genet. A, 126, 221-228 (2004).

12) Kamatani Y., Matsuda K., Ohishi T., Ohtsubo S., Yamazaki K., Iida A., Hosono N., Kubo M., Yumura W., Nitta K., Katagiri T., Kawaguchi Y., Kamatani N., Nakamura Y., J. Hum. Genet., 53, 64-73 (2008).

13) Minamitani T., Ikuta T., Saito Y., Takebe G., Sato M., Sawa H., Nishimura T., Nakamura F., Takahashi K., Ariga H., Matsumoto K., Exp. Cell Res., 298, 305-315 (2004).

14) Egging D., van den Berkmortel F., Taylor G., Bristow J., Schalkwijk J., Arch. Dermatol. Res., 298, 389-396 (2007).

15) Veit G., Hansen U., Keene D. R., Bruckner P., Chiquet-Ehrismann R., Chiquet M., Koch M., J. Biol. Chem., 281, 27461-27470 (2006).

16) Zweers M. C., van Vlijmen-Willems I. M., van Kuppevelt T. H., Mecham R. P., Steijlen P. M., Bristow J., Schalkwijk J., J. Invest. Dermatol., 122, 885-891 (2004).

17) Lethias C., Carisey A., Comte J., Cluzel C., Exposito J. Y., FEBS Lett., 580, 6281-6285 (2006).

18) Elefteriou F., Exposito J. Y., Garrone R., Lethias C., FEBS Lett., 495, 44-47 (2001).

19) Zweers M. C., Peeters A. C., Graafsma S., Kranendonk S., van der Vliet J. A., den Heijer M., Schalkwijk J., Circulation, 113, 1702-
1707 (2006).

20) Matsumoto K., Kinoshita T., Hirose T., Ariga H., DNA Cell Biol., 25, $448-456$ (2006).

21) O'Reilly M. S., Holmgren L., Shing Y., Chen C., Rosenthal R. A., Moses M., Lane W. S., Cao Y., Sage E. H., Folkman J., Cell, 79, 315328 (1994).

22) O'Reilly M. S., Boehm T., Shing Y., Fukai N., Vasios G., Lane W. S., Flynn E., Birkhead J. R., Olsen B. R., Folkman J., Cell, 88, 277-285 (1997).

23) Kamphaus G. D., Colorado P. C., Panka D. J., Hopfer H., Ramchandran R., Torre A., Maeshima Y., Mier J. W., Sukhatme V. P., Kalluri R., J. Biol. Chem., 275, 1209-1215 (2000).

24) Maeshima Y., Sudhakar A., Lively J. C., Ueki K., Kharbanda S., Kahn C. R., Sonenberg N., Hynes R. O., Kalluri R., Science, 295, 140-143 (2002).

25) Yi M., Ruoslahti E., Proc. Natl. Acad. Sci. U.S.A., 98, 620-624 (2001).

26) Mongiat M., Sweeney S. M., San Antonio J. D., Fu J., Iozzo R. V., J. Biol. Chem., 278, 4238-4249 (2003).

27) Ikuta T., Ariga H., Matsumoto K., Genes Cells, 5, 913-927 (2000).

28) Ikuta T., Sogawa N., Ariga H., Ikemura T., Matsumoto K., Gene, 217, $1-13$ (1998).

29) Minamitani T., Ariga H., Matsumoto K., Biochem. Biophys. Res. Commun., 267, 626-631 (2000).

30) Suhardja A., Hoffman H., Microsc. Res. Tech., 60, 70-75 (2003).

31) Elefteriou F., Exposito J. Y., Garrone R., Lethias C., Eur. J. Biochem., 263, 840-848 (1999).

32) Lethias C., Elefteriou F., Parsiegla G., Exposito J. Y., Garrone R., J. Biol. Chem., 276, 16432-16438 (2001). 